\title{
Risk Factors of Asymptomatic Neurocognitive Impairment in People Living with HIV in an Indian Cohort
}

\author{
Salil Gupta ${ }^{1}$ Nirmala Venugopal² \\ ${ }^{1}$ Department of Neurology, Army Hospital Research and Referral, \\ New Delhi, India \\ 2Department of Internal Medicine, Military Hospital Bagdogra, \\ Darjeeling District, West Bengal, India
}

\begin{abstract}
Address for correspondence Salil Gupta, MD, DNB, DM, Department of Neurology, Army Hospital Research and Referral, Delhi Cantonment, New Delhi-110010, India (e-mail: chickusalil@yahoo.com).
\end{abstract}

J Neurosci Rural Pract 2020;11:230-236

\begin{abstract}
Keywords

- asymptomatic

- cognition

- education

- gender

- HIV

- risk factor

- vascular

Background Asymptomatic neurocognitive impairment (ANI) in people living with HIV (PLWH) can lower quality of life, reduce drug compliance, increase unemployment, and reduce life expectancy.

Objective This study was aimed to identify risk factors of ANI in PLWH in an Indian cohort and explore the usefulness of Mini Mental State Examination (MMSE) and Montreal Cognitive Assessment Score (MoCA) as screening tools.

Methods PLWH under follow-up at an antiretroviral treatment center who were 18 to 60 years were included in this study. Patients were excluded if they had any cognitive symptoms, previous history of any central nervous system (CNS) pathology, or any systemic illness. Included patients were subjected to domain wise standardized neuropsychological battery. Six domains were screened including language, attention, speed, memory, sensory motor skills, and executive. Abnormal dysfunctional scores in at least two domains were taken as suggestive of ANI. The two groups thus created, ANI and normal cognition, were evaluated for differences. Variables evaluated as risk factors included age, sex, handedness, education, presence of at least one vascular risk factor, duration of disease, biochemical profile, cluster of differentiation 4 (CD4) count (both current and nadir) HIV viral load, and use of antiretroviral therapy (ART) and its CNS penetration effectiveness (CPE). MMSE and MoCA were also done in all patients.

Statistical Analysis Regression analysis was used to find out significant variables. MMSE and MoCA scores were correlated using Spearman's correlation coefficient. Sensitivity, specificity, positive predictive value (PPV), and negative predictive value (NPV) were also determined

Results Three hundred and eighty-four patients were included out of which 185 (48\%) had ANI. In the multivariate regression analysis, female sex with odds Ratio (OR) of 1.89 (95\% confidence interval $[\mathrm{Cl}]: 1.21-2.79, p<0.01$ ), education below 10 years with $\mathrm{OR}=2.43$ $(95 \% \mathrm{Cl}: 1.56-3.80, p<0.01)$ and presence of at least one vascular risk factor with $\mathrm{OR}=2.52$ (95\% Cl: $1.67-3.80, p<0.01$ ) were found to be significant. Both MMSE and MoCA had a high PPV (0.99 and 0.97, respectively) but poor NPV (0.64 and 0.75 ) below a score of 25 with MoCA scoring slightly better. Both, MMSE and MoCA correlated well with each other.

Conclusion Nearly half of our patients had ANI, despite being on ART. Majority of patients were on ART with CPE $>7$ and had relatively preserved immune status. Female HIV patients with at least one vascular risk factor and less than 10 years of formal education were found to be at risk for ANI. MMSE and MoCA are not good screening tools to identify this condition.
\end{abstract}

License terms

() (1) $\ominus \circledast$ 


\section{Introduction}

Changes in attention, memory, concentration, and motor skills are common in people living with HIV (PLWH). This neurocognitive impairment (NI) presents a diagnostic challenge to the clinician. Such a presentation can be caused by a variety of disorders including infections, drugs, metabolic causes, substance abuse, prior head trauma, and HIV itself. Mostly, a detailed evaluation should be able to exclude common causes. Neurocognitive impairment not clearly attributable to an alternate cause other than HIV infection has been collectively classified as HIV-associated neurocognitive disorders (HAND).

The terminology of HAND has evolved over time. A working group supported by the United States National Institutes of Health published a classification scheme in 2007 for diagnostic and research purposes. ${ }^{1}$ This classification, known as the "Frascati criteria," has been widely adopted now, although with a few dissenting voices. ${ }^{2}$ HAND includes three levels of impaired performance on standardized neuropsychological tests and functional impairment. Asymptomatic neurocognitive impairment (ANI) is defined as one standard deviation or more below the mean in at least two cognitive domains without symptomatic or functional impairment. Mild neurocognitive disorder (MND) is said to be present if the individual scores 1 standard deviation or more below the mean in at least two cognitive domains with at least mild symptomatic or functional impairment. HIV-associated dementia (HAD) is defined by a score of 2 standard deviations or more below the mean in at least two cognitive domains with impairment in activities of daily living. It is important to note that two individuals may have a similar score of 1 standard deviation below the mean, but it is the presence or absence of symptoms and functional impairment which will characterize them further to MND or ANI respectively. For example, a high-performing multitasking marketing executive may report with poor concentration and inability to perform at work as earlier. He scores 1 standard deviation below the mean on standardized neuropsychological tests and is said to have MND. An asymptomatic manual laborer, who undergoes the same battery, scores like the first individual but is labeled as ANI.

Neurocognitive impairment is commonly reported in PLWH and is likely to become an increasingly important issue as this population ages. HIV-associated dementia, which occurred in up to $50 \%$ of patients prior to the introduction of antiretroviral therapy (ART), is now rare. However, the milder forms, ANI and MND, are more widely reported and may occur in as much as half of the population of PLWH. ${ }^{3,4,5,6}$ This results in lower quality of life, poorer adherence to medication, increased unemployment, and reduced life expectancy. ${ }^{7-9}$ ANI is clinically relevant, as it can transform into the more severe forms of HAND as documented in the CHARTER (CNS HIV Antiretroviral Therapy Effects Research) study. ${ }^{10}$ It is therefore important to identify the risk factors of ANI early and possibly intervene to prevent progression. The risk factors appear to be changing in the ART era. Previous studies have suggested that cardiovascular risk factors, age, hepatitis
C infection, cluster of differentiation 4 (CD4) count nadir, addictions, previous psychiatric, or neurological disorders may contribute to the development of HAND. However, these risk factors are not well defined in India and in the developing world. ART has changed the profile of HAND in India with milder ANI and MND being more common. ${ }^{11}$ But the risk factors leading to the development of HAND, especially ANI remain elusive. If the risk factors of HAND, especially ANI can be identified early and controlled, then the long-term effects of poor cognition can be limited. The aim of this study was to identify the risk factors associated with the development of ANI in PLWH in India.

\section{Patients and Methods}

This cross-sectional observational study was conducted at a government run ART center of a medical college in a metropolis in India. Patients were recruited over a period of 2 years. The inclusion criteria included HIV infected patients between 18 and 60 years of age who were registered with the ART center. Patients were excluded if they or their caregivers gave history suggestive of cognitive decline; there was history of past or present neurological infections, central nervous system (CNS) pathology like neoplasm, stroke or degenerative diseases, significant head trauma, defined as loss of consciousness for more than 5 minutes or development of retrograde or antegrade amnesia, epilepsy; any history of being treated for a psychiatric illness including depression and substance abuse or alcohol dependence syndrome. Patients were also excluded if they were being currently treated for any other systemic illness related to HIV like opportunistic infection or neoplasm or systemic diseases like thyroid, liver, or renal disease.

All patients were subjected to a battery of neuropsychological tests by the second author. She in turn had been trained to administer the tests by a senior clinical psychologist from the same institute. The licensed battery (available at the institute) included standard, validated tests designed to evaluate a particular domain(s) of cognition. These were Verbal Adult Intelligence Scale (VAIS; Language), Postgraduate Institute (PGI, in Chandigarh) memory scale, attention subset (attention), PGI memory scale (memory) and Bender Gestalt test (sensory motor skill), and PGI battery of brain dysfunction (PGI-BBD). ${ }^{12}$ Trail making test A and B (speed) and Wisconsin card sorting test (execution) were also included. To capitalize on the previously available normative data, the raw scores were converted into demographically corrected scores using a norms table given with the tool. Utilizing a screener that does account for certain demographic factors (age, education, and ethnicity) is inappropriate when these variables are known to affect cognitive functioning. To obviate bias a dysfunction rating score was used to compare scores across education and age. If at least in two cognitive domains patient had higher dysfunction scores, as defined by the cut-offs provided with the tool, patients were categorized into having "HIV-associated ANI." If the dysfunction scores were normal in all domains or abnormal in one, then the patient was categorized as having "HIV infection with no 
cognitive impairment." The two groups thus created were then compared for differences in defined variables.

All patients had details recorded like age, sex, handedness, years of education, presence (or absence) of at least one vascular risk factor, and total duration of disease. The vascular risk factors included tobacco use (in any form), hypertension, diabetes, heart disease, or family history of premature coronary artery disease, stroke, or dementia. Depending on the number of years of formal education the patients were divided into three groups: less than 5 years, 5 to 10 years, and more than 10 years of education. Blood tests like hemoglobin, sugar, lipid profile, current and nadir CD4 count, and HIV viral load where available were also recorded. If they were taking ART, then the type of combination and the CNS penetration effectiveness (CPE) of the ART were also recorded. These variables were compared between those having abnormal and normal cognition as risk factor of ANI. In addition, Mini Mental Scale Examination (MMSE) and Montreal Cognitive Assessment (MOCA) scores were also determined for each patient and compared between the two groups for statistical difference. A cut-off of $<25$ was assumed as abnormal for both.

The sample size for estimating difference between proportions in population was calculated to be 184 for each arm keeping the $\alpha$ error to be 0.05 , power of study as $80 \%$, absolute error of margin of $10 \%$ and assuming from previous studies that around $40 \%$ of patients are likely to have some form of neurocognitive dysfunction. The data were analyzed using IBM SPSS 22 software. The two created groups of ANI and normal cognition in HIV infected patients were compared using univariate analysis using appropriate tests as mentioned in the tables. Multivariate regression analysis was done on significant variables. MMSE and MoCA scores were also correlated for each of the groups using Spearman's Correlation Coefficient. Sensitivity, specificity, positive predictive value (PPV), and negative predictive value (NPV) of both MMSE and MoCA were also determined. A $p$-value of $<0.05$ was taken as significant. Written informed consent was taken from all patients and ethical clearance from institute ethics committee.

\section{Results}

A total of 384 patients were included in the study out of which 185 were diagnosed to have HIV infection with ANI and 199 had HIV infection with normal cognition. Univariate analysis for various risk factors using appropriate statistical test are shown in - Table $\mathbf{1}$. Variables found to be significant

Table 1 Univariate analysis of risk factors of ANI in HIV patients

\begin{tabular}{|c|c|c|c|c|c|}
\hline Sr. no. & Variables & $\begin{array}{l}\text { HIV infection with } \\
\text { asymptomatic } \\
\text { neurocognitive } \\
\text { impairment }(n=185 \text { ) }\end{array}$ & $\begin{array}{l}\text { HIV infection with } \\
\text { normal cognition } \\
(n=199)\end{array}$ & Test & $p$-Value \\
\hline 1 & Age in years (SD) & $38.3(9.3)$ & $38.3(9.1)$ & Mann-Whitney U-test & 0.68 \\
\hline 2 & Females (\%) & $83(44.9)$ & $61(30.7)$ & $\begin{array}{l}z \text {-Score for two population } \\
\text { proportion }\end{array}$ & $<0.01$ \\
\hline \multirow[t]{4}{*}{3} & Education in years (\%) & & & \multirow{4}{*}{$\begin{array}{l}z \text {-Score for two population } \\
\text { proportion }\end{array}$} & \\
\hline & $<5$ & $68(36.8)$ & $61(30.7)$ & & 0.2 \\
\hline & $5-10$ & $75(40.5)$ & $55(27.6)$ & & $<0.01$ \\
\hline & $>10$ & $42(22.7)$ & $83(41.7)$ & & $<0.01$ \\
\hline 4 & Righthanded (\%) & $164(88.6)$ & $186(93.5)$ & $\begin{array}{l}z \text {-Score for two population } \\
\text { proportion }\end{array}$ & 0.09 \\
\hline \multirow[t]{4}{*}{5} & $\begin{array}{l}\text { Presence of at least one } \\
\text { vascular cognitive risk } \\
\text { factor (\%) }\end{array}$ & & & $\begin{array}{l}z \text {-Score for two population } \\
\text { proportion }\end{array}$ & \\
\hline & No risk factor & $76(41.1)$ & $127(63.8)$ & & $<0.01$ \\
\hline & 1 risk factor & $86(46.5)$ & $60(30.2)$ & & $<0.01$ \\
\hline & $\geq 2$ risk factor & $23(12.4)$ & $12(6.0)$ & & 0.02 \\
\hline 6 & Hemoglobin in $\mathrm{g} / \mathrm{dL}(\mathrm{SD})$ & $12.4(1.6)$ & $12.2(1.7)$ & Mann-Whitney U-test & 0.29 \\
\hline 7 & $\begin{array}{l}\text { Blood sugar fasting in } \\
\mathrm{mg} / \mathrm{dL}(\mathrm{SD})\end{array}$ & $95.4(9.1)$ & $94.3(8.4)$ & Mann-Whitney U-test & 0.20 \\
\hline 8 & $\begin{array}{l}\text { Blood sugar postprandial } \\
\text { in } \mathrm{mg} / \mathrm{dL}(\mathrm{SD})\end{array}$ & $152.4(15.2)$ & $150.1(15.4)$ & Mann-Whitney U-test & 0.10 \\
\hline 9 & $\begin{array}{l}\text { Total cholesterol in } \\
\mathrm{mg} / \mathrm{dL} \text { (SD) }\end{array}$ & $197.4(33.9)$ & $196.2(33.2)$ & Mann-Whitney U-test & 0.68 \\
\hline 10 & LDL in mg/dL (SD) & $128.8(33.9)$ & $127.1(32.5)$ & Mann-Whitney U-test & 0.55 \\
\hline 11 & $\mathrm{HDL}$ in $\mathrm{mg} / \mathrm{dL}$ (SD) & $37.8(5.0)$ & $39.1(6.0)$ & Mann-Whitney U-test & 0.06 \\
\hline
\end{tabular}


Table 1 (continued)

\begin{tabular}{|c|c|c|c|c|c|}
\hline Sr. no. & Variables & $\begin{array}{l}\text { HIV infection with } \\
\text { asymptomatic } \\
\text { neurocognitive } \\
\text { impairment }(n=185)\end{array}$ & $\begin{array}{l}\text { HIV infection with } \\
\text { normal cognition } \\
(n=199)\end{array}$ & Test & $p$-Value \\
\hline 12 & $\begin{array}{l}\text { Triglycerides in } \mathrm{mg} / \mathrm{dL} \\
\text { (SD) }\end{array}$ & $150.9(28.3)$ & $149.3(27.9)$ & Mann-Whitney U-test & 0.60 \\
\hline 13 & CD4 count nadir/ $\mu \mathrm{L}(\mathrm{SD})$ & $143.7(132.6)$ & $177.4(134.6)$ & Mann-Whitney U-test & $<0.01$ \\
\hline 14 & $\begin{array}{l}\text { CD4 count present/cm } \\
\text { (SD) }\end{array}$ & $393.8(238.1)$ & $448.7(235.5)$ & Mann-Whitney U-test & $<0.01$ \\
\hline 15 & $\begin{array}{l}\text { Median serum HIV RNA } \\
\text { copies/mL (IQR) }\end{array}$ & $\begin{array}{l}(n=76) \\
68,839(9,716,429)\end{array}$ & $\begin{array}{l}(n=38) \\
105,805(848,286)\end{array}$ & Mann-Whitney U-test & 0.41 \\
\hline 16 & $\begin{array}{l}\text { Total duration of disease } \\
\text { in years (SD) }\end{array}$ & $5.7(3.4)$ & $6.1(3.4)$ & Mann-Whitney U-test & 0.16 \\
\hline 17 & $\begin{array}{l}\text { Number of patients on } \\
\text { ART (\%) }\end{array}$ & $185(100)$ & 159 (79.9) & $\begin{array}{l}z \text {-Score for two population } \\
\text { proportion }\end{array}$ & $<0.01$ \\
\hline 18 & Median CPE of ART (SD) & $10(1.8)$ & $10(3.9)$ & & \\
\hline 19 & $\begin{array}{l}\text { Number of patients on } \\
\text { ART with CPE of }>7(\%)\end{array}$ & $128(69.1)$ & $131(65.8)$ & $\begin{array}{l}z \text {-Score for two population } \\
\text { proportion }\end{array}$ & 0.48 \\
\hline
\end{tabular}

Abbreviations: ANI, asymptomatic neurocognitive impairment; ART, antiretroviral therapy; CD4, cluster of differentiation 4; CNS, central nervous system; CPE, CNS penetration effectiveness; HDL, high-density lipoprotein; HIV, human immunodeficiency virus; IQR, interquartile range; LDL, low-density lipoprotein; SD, standard deviation;

including female sex, education levels below 10 years, presence of at least one vascular risk factor, CD4 count present, and nadir were included in the multivariate regression analysis. In the multivariate regression analysis female sex with odds ratio (OR) of 1.89 (95\% confidence interval [CI]: $1.21-2.79, p<0.01$ ), education below 10 years with $\mathrm{OR}=2.43$ (95\% CI: $1.56-3.80, p<0.01$ ), and presence of at least one vascular risk factor with $\mathrm{OR}=2.52(95 \% \mathrm{CI}: 1.67-3.80, p<0.01)$ were found to be significant (-Table 2). Although there were more patients on ART in the ANI group, the proportion of patients taking ART with CPE $>7$ was similar in both the groups. The median CPE was also similar in both groups.

- Table 3 shows the number and percentage of patients who had MMSE and MoCA scores $<25$. There was significant correlation of overall scores of MMSE with MoCA in both categories, ANI $(0.77,<0.01)$ and normal cognition patients $(0.84,<0.01)$ ( - Table 4$)$. This is also depicted in the form of a box plot in - Fig. 1. Both MMSE and MoCA, for scores $<25$, had a high-PPV for predicting cognitive impairment but poor NPV with MoCA scoring a little better than MMSE ( - Table 5).

\section{Discussion}

Over the last few years, the widespread use of combination antiretroviral therapy has been associated with a marked decrease in the incidence of more severe neurocognitive deficits (HAD). ${ }^{13}$ However, this is not true with respect to milder forms of NI where studies have shown the prevalence to have ranged from 30 to $50 \%^{3-6}$ Our study has shown that nearly half ( $48 \%$ ) of our cohort of HIV infected patients, who were cognitively asymptomatic (self-reported), had significantly abnormal scores in at least two of six domains tested on a standardized neuropsychological battery. This is despite the fact that all 185 patients (100\%) in the ANI group

Table 2 Multivariate regression analysis of risk factors of ANI in HIV patients

\begin{tabular}{|c|c|c|c|c|c|}
\hline \multicolumn{6}{|c|}{ Coefficients } \\
\hline \multirow[t]{2}{*}{ Model } & \multicolumn{2}{|c|}{ Unstandardized coefficients } & \multirow{2}{*}{$\begin{array}{l}\text { Standardized } \\
\text { coefficients } \\
\text { Beta }\end{array}$} & \multirow[t]{2}{*}{$t$-Test } & \multirow[t]{2}{*}{ Sig. } \\
\hline & B & SE & & & \\
\hline (Constant) & 1.251 & 0.800 & & 1.564 & 0.121 \\
\hline Sex & -0.224 & 0.105 & -0.236 & -2.133 & 0.036 \\
\hline Education & 0.120 & 0.057 & 0.210 & 2.090 & 0.039 \\
\hline $\begin{array}{l}\text { Presence of at least one } \\
\text { vascular risk factors }\end{array}$ & -0.286 & 0.076 & -0.439 & -3.755 & 0.000 \\
\hline CD4 nadir & 0.000 & 0.001 & 0.028 & 0.287 & 0.775 \\
\hline CD4 present & 0.001 & 0.000 & 0.135 & 1.287 & 0.201 \\
\hline
\end{tabular}

Abbreviations: CD4, cluster of differentiation 4; SE, standard error.

$B$ or Beta is a value of the statistical test of coefficients and sig. stands for significance $(p$-value $<0.05)$ 
Table 3 Distribution of patients as per their MMSE and MoCA scores

\begin{tabular}{|l|l|l|}
\hline Test $(\boldsymbol{n}=\mathbf{3 8 4})$ & Scores $<25(\%)$ & Scores $\geq 25(\%)$ \\
\hline MMSE & $77(20.1)$ & $307(79.9)$ \\
\hline MoCA & $123(32)$ & $261(68)$ \\
\hline
\end{tabular}

Abbreviations: MMSE, mini mental state examination; MoCA, Montreal cognitive assessment score.

Table 4 Correlation between MMSE and MoCA

\begin{tabular}{|l|l|l|l|l|}
\hline & $\begin{array}{l}\text { Mean } \\
\text { MMSE }\end{array}$ & $\begin{array}{l}\text { Mean } \\
\text { MoCA }\end{array}$ & $\begin{array}{l}\text { Spearman's } \\
\text { Correlation Coefficient }\end{array}$ & $p$-Value \\
\hline $\begin{array}{l}\text { No } \\
\text { ANI }\end{array}$ & 29.04 & 28.49 & 0.77 & $<0.01$ \\
\hline ANI & 25.01 & 22.73 & 0.84 & $<0.01$ \\
\hline
\end{tabular}

Abbreviations: ANI, asymptomatic neurocognitive impairment; MMSE, mini mental state examination; MoCA, Montreal cognitive assessment score. were on ART and were virally suppressed with relatively preserved CD4 counts. Our study highlights a critical issue which has not been resolved as yet. Is this milder form of $\mathrm{NI}$ in virally suppressed individuals the result of subclinical brain injury sustained before starting ART? There have also been concerns raised earlier about the neurotoxicity of ART, especially those with greater CNS penetration..$^{14}$ Could this also contribute to the milder forms of NI seen more often now? Although there was no significant difference in the proportion of patients with CPE greater than 7 in the two groups, it is possible that drugs may have contributed to the NI in a previously injured brain.

Multivariate analysis revealed that the risk factors for development of ANI in our cohort were female sex with OR of 1.89 (95\% CI: $1.21-2.79, p<0.01$ ), education below 10 years with OR $=2.43(95 \% \mathrm{CI}: 1.56-3.80, p<0.01)$ and presence of at least one vascular risk factor with OR 2.52 (95\% CI: 1.67-3.80, $p<0.01$ ). Our results are similar to multicenter AIDS cohort

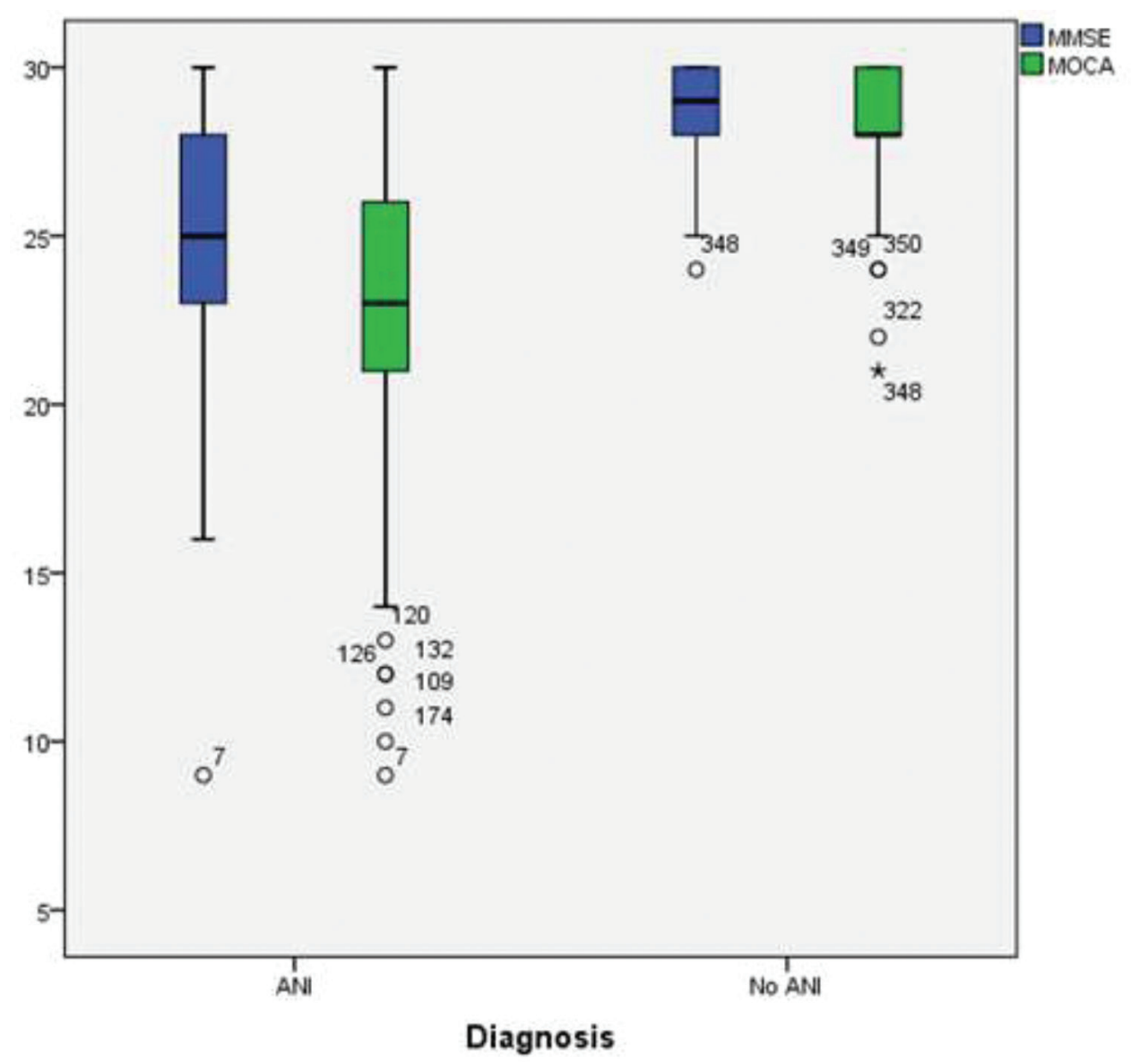

Fig. 1 Box plot showing correlation of MMSE and MoCA in patients with ANI and normal cognition. ANI, asymptomatic neurocognitive impairment; MMSE, mini mental state examination; MoCA, Montreal cognitive assessment score.

Table 5 Sensitivity, specificity, positive predictive value and negative predicitive value of MMSE and MoCA

\begin{tabular}{|l|l|l|l|l|}
\hline & Sensitivity $(\mathrm{Cl})$ & Specificity & PPV & NPV \\
\hline MMSE $<25$ & $0.41(0.34-0.48)$ & $0.99(0.98-1.0)$ & 0.99 & 0.64 \\
\hline MoCA $<25$ & $0.64(0.57-0.71)$ & $0.98(0.96-1.0)$ & 0.97 & 0.75 \\
\hline
\end{tabular}

Abbreviations: $\mathrm{Cl}$, confidence interval; MMSE, mini mental state examination; MoCA, Montreal cognitive assessment score; NPV, negative predictive value; PPV, positive predictive value. 
study (MACS) cohort which suggests that higher education is protective against HAND. ${ }^{15}$ Female sex was found to be a risk factor in our study. This finding has not been seen in any other previous study and needs to be validated. Vascular risk factors were linked to poorer cognitive performance in the MACS. ${ }^{16}$ Central obesity and diabetes were important risk factors for HAND in the CHARTER cohort. ${ }^{17}$ We did not evaluate central obesity. In a study of 245 HIV infected patients in Italy, diabetes, carotid intimamedia thickness (CIMT), and vascular risk factors, including hyperlipidemia and tobacco use, were strongly associated with lower cognitive performance. ${ }^{18}$ Diabetes has also been linked to greater cognitive decline a French cohort of HIV patients. ${ }^{19}$ Our previous study has shown that CIMT is linked to protease inhibitor based ART regiemes. ${ }^{20}$ Previous studies have shown that age, especially $>50$ years, increases risk of HAND. ${ }^{21,22}$ However we did not find a similar association possibly because our population was younger. Also, in the previous cohorts presence of vascular risk factors in the older patients may have confounded the results.

One of the objectives of our study was to test the usefulness of using popular cognitive screening tools like MMSE and MoCA in HIV patients with ANI. They were chosen despite their well-known inherent drawbacks in view of their familiarity with most physicians and ease of administration in a busy OPD. We did not use Hindi or any other regional language MMSE. We found that if a score of $<25$ is taken as abnormal then both MMSE and MoCA have a high PPV but poor NPV for ANI, although MoCA fared a little better than MMSE. They are not good tools to screen for cognitive impairment in asymptomatic individuals. This is similar to previous studies. ${ }^{23,24}$ This makes them poor screening tools.

Surprisingly, we did not find an association with CD4 count (current and nadir) and HIV viral loads. This is similar to another study by Heaton et al who found HAD to occur with less immunosuppression..$^{25}$ However CD4 count nadir was strongly linked to cognitive impairment in a study by Ellis et al. ${ }^{26}$ Our study could not replicate the same results in multivariate regression analysis. This could be related to the type of patients we included in the study (only asymptomatic patients).

There are several limitations to our study. They include lack of brain imaging, especially of those who were detected to have ANI. Also, we did not screen for vitamin B12 deficiency and thyroid disorders after detecting ANI. Our diagnosis of ANI was based on self-reporting of no cognitive symptoms where a bias was highly possible. We did not include several vascular risk factors like obesity, homocysteine levels, etc. Less than a third our patients had HIV viral load levels. Data on duration of ART was not available and may have contributed to bias, although factors governing effect of ART on cognition like CPE were taken into consideration. Finally, this was a cross-sectional study.

\section{Conclusion}

In conclusion, we found that nearly half of our cohort of HIV patients who were asymptomatic had neurocognitive impairment. This is despite the fact that nearly all patients were on ART with CPE of $>7$ and were relatively immune preserved. Female HIV patients with at least one vascular risk factor and less than 10 years of formal education are most at risk for asymptomatic neurocognitive impairment. We have raised concerns that this mild NI may be the result of subclinical brain injury by HIV, possibly prior to starting ART. A standardized neuropsychological battery is needed to diagnose this condition. MMSE and MoCA are may not good screening tools.

What is the translational value of this study? HIV physicians should be aware of the possibility of ANI and mild neurocognitive impairment despite the patient being on ART. They should be particularly alert in the high-risk group described in our study. Diagnosis can be established only by neuropsychological evaluation using a standardized battery. Such high-risk asymptomatic patients, with any one of the significant risk factors seen in our study, should be referred for detailed neuropsychological evaluation. Counselling and managing the vascular risk factor may partially mitigate the risk. Further research is needed to explore if starting ART at diagnosis reduces the prevalence of milder forms of NI.

\section{Note}

The authors certify that this research has no sponsoring authority. This was a self-driven research.

\section{Ethical Approval}

Institutional Ethics review committee approval from Armed Forces Medical College has been taken for this study.

\section{Funding}

None.

\section{Conflict of Interest}

None declared.

\section{References}

1 Antinori A, Arendt G, Becker JT, et al. Updated research nosology for HIV-associated neurocognitive disorders. Neurology 2007;69(18):1789-1799

2 Gisslén M, Price RW, Nilsson S. The definition of HIV-associated neurocognitive disorders: are we overestimating the real prevalence? BMC Infect Dis 2011;11:356

3 Simioni S, Cavassini M, Annoni JM, et al. Cognitive dysfunction in HIV patients despite long-standing suppression of viremia. AIDS 2010;24(9):1243-1250

4 Heaton RK, Clifford DB, Franklin DR Jr, et al; CHARTER Group. HIV-associated neurocognitive disorders persist in the era of potent antiretroviral therapy: CHARTER Study. Neurology 2010;75(23):2087-2096

5 Sacktor N, Skolasky RL, Seaberg E, et al. Prevalence of HIVassociated neurocognitive disorders in the multicenter AIDS cohort study. Neurology 2016;86(4):334-340

6 Pumpradit W, Ananworanich J, Lolak S, et al; Southeast Asia Research Collaboration with Hawaii (SEARCH) 005 Protocol Team. Neurocognitive impairment and psychiatric comorbidity in well-controlled human immunodeficiency virus-infected Thais from the 2NN Cohort Study. J Neurovirol 2010;16(1):76-82 
7 Morgan EE, Woods SP, Grant I; HIV Neurobehavioral Research Program (HNRP) Group. Intra-individual neurocognitive variability confers risk of dependence in activities of daily living among HIV-seropositive individuals without HIVassociated neurocognitive disorders. Arch Clin Neuropsychol 2012;27(3):293-303

8 Woods SP, Weber E, Weisz BM, Twamley EW, Grant I; HIV Neurobehavioral Research Programs Group. Prospective memory deficits are associated with unemployment in persons living with HIV infection. Rehabil Psychol 2011;56(1):77-84

9 Gorman AA, Foley JM, Ettenhofer ML. Hinkin CH, van Gorp WG. Functional consequences of HIV-associated neuropsychological impairment. Neuropsychol Rev 2009;19(2):186-203

10 Grant I, Franklin DR Jr, Deutsch R, et al; CHARTER Group. Asymptomatic HIV-associated neurocognitive impairment increases risk for symptomatic decline. Neurology 2014;82(23):2055-2062

11 Ghate M, Mehendale S, Meyer R, et al. The effects of antiretroviral treatment initiation on cognition in HIV-infected individuals with advanced disease in Pune, India. J Neurovirol 2015;21(4):391-398

12 Pershad D, Verma SK, Handbook of PGI Battery of Brain Dysfunction. Agra, India: National Psychological Corp.; 2009

13 Bhaskaran K, Mussini C, Antinori A, et al; CASCADE Collaboration. Changes in the incidence and predictors of human immunodeficiency virus-associated dementia in the era of highly active antiretroviral therapy. Ann Neurol 2008;63(2):213-221

14 Robertson K, Liner J, Meeker RB. Antiretroviral neurotoxicity. J Neurovirol 2012;18(5):388-399

15 Becker JT, Kingsley LA, Molsberry S, et al. Cohort profile: recruitment cohorts in the neuropsychological substudy of the multicenter AIDS cohort study. Int J Epidemiol 2015;44(5):1506-1516

16 Becker JT, Kingsley L, Mullen J, et al; Multicenter AIDS Cohort Study. Vascular risk factors, HIV serostatus, and cognitive dysfunction in gay and bisexual men. Neurology 2009;73(16):1292-1299

17 McCutchan JA, Marquie-Beck JA, Fitzsimons CA, et al; CHARTER Group. Role of obesity, metabolic variables, and diabetes in HIV-associated neurocognitive disorder. Neurology 2012;78(7):485-492

18 Fabbiani M, Ciccarelli N, Tana M, et al. Cardiovascular risk factors and carotid intima-media thickness are associated with lower cognitive performance in HIV-infected patients. HIV Med 2013;14(3):136-144

19 Dufouil C, Richert L, Thiébaut R, et al; ANRS CO3 Aquitaine Study Group. Diabetes and cognitive decline in a French cohort of patients infected with HIV-1. Neurology 2015;85(12):1065-1073

20 Salil G, Manish B, Velu N, Tewari AK, Nardeep N. Accelerated subclinical atherosclerosis in human immunodeficiency virus-infected patients on protease inhibitor regime. J HIV Hum Reprod 2014;2:48-53

21 Fazeli PL, Crowe M, Ross LA, Wadley V, Ball K, Vance DE. Cognitive functioning in adults aging with HIV: a cross-sectional analysis of cognitive subtypes and influential factors. J Clin Res HIV AIDS Prev 2014;1(4):155-169

22 Valcour V, Shikuma C, Shiramizu B, et al. Higher frequency of dementia in older HIV-1 individuals: the Hawaii Aging with HIV-1 Cohort. Neurology 2004;63(5):822-827

23 Milanini B, Wendelken LA, Esmaeili-Firidouni P, Chartier M, Crouch PC, Valcour V. The Montreal cognitive assessment to screen for cognitive impairment in HIV patients older than 60 years. J Acquir Immune Defic Syndr 2014;67(1):67-70

24 Skinner S, Adewale AJ, DeBlock L, Gill MJ, Power C. Neurocognitive screening tools in HIV/AIDS: comparative performance among patients exposed to antiretroviral therapy. HIV Med 2009;10(4):246-252

25 Heaton RK, Franklin DR, Ellis RJ, et al; CHARTER Group; HNRC Group. HIV-associated neurocognitive disorders before and during the era of combination antiretroviral therapy: differences in rates, nature, and predictors. J Neurovirol 2011;17(1):3-16

26 Ellis RJ, Badiee J, Vaida F, et al; CHARTER Group. CD4 nadir is a predictor of HIV neurocognitive impairment in the era of combination antiretroviral therapy. AIDS 2011;25(14):1747-1751 